\title{
Thin-Layer Chromatographic Identification of Chain Architectures of Styrene-Butadiene Copolymers
}

\author{
Nobuo Donkai, ${ }^{*}$ Takeaki Miyamoto, and Hiroshi Inagaki** \\ Institute for Chemical Research, Kyoto University, \\ Uji, Kyoto 611, Japan.
}

(Received May 19, 1975)

\begin{abstract}
The feasibilities of thin-layer chromatography as a tool to identify the chain architecture of given copolymer samples have been investigated. To this end, styrene-butadiene (SB) block copolymers with known chain architecture were prepared and used as reference samples. Preliminary TLC experiments on these samples indicated that the random, tapered, diblock and triblock SB-samples could be chromatographically distinguished from one another under suitable development conditions. By applying the procedures thus established, the chain architecture of various commercial SB-products could be identified without appreciable interference from the molecular weight and composition.
\end{abstract}

KEY WORDS Thin-Layer Chromatography / Adsorption Chromatography / Precipitation Chromatography / Styrene-Butadiene Copolymer / Block Copolymer / Tapered Copolymer / Chain Architecture /

In one of our previous papers ${ }^{1}$ it was reported that styrene-methyl methacrylate (SM) copolymers having the same composition but different chain architectures could be distinguished from one another with thin-layer chromatography (TLC). On the chromatograms obtained, the random and alternating polymer both showed intermediate values of $R f$ (rate of flow) whereas the block polymer remained immobile on the starting point. Recently we observed further that the TLC behavior of SM-diblock polymers differed distinctly from that of MSM-triblock polymers even on the same composition level. ${ }^{2}$ The above two findings were attained using a separation mechanism in TLC, which involves adsorption-desorption processes. $^{3}$ Since this mechanism has been known to lead to polymer separation without appreciable interference from the molecular weight, ${ }^{3}$ we thought it feasible with TLC to identify the chain architecture of given block-copolymer samples independent of their molecular weight. This paper deals with such identification problems for styrenebutadiene (SB) copolymers which are now of

* Present address: Midland Macromolecular Institute, Midland, Michigan 48640, USA.

** To whom correspondence should be addressed. industrial interest. ${ }^{4}$

\section{EXPERIMENTAL}

\section{Sample Polymers}

Three reference samples of SB-block copolymers having different chain architectures were prepared by the anionic polymerization technique. ${ }^{5}$ Each polymerization run was conducted at $30^{\circ} \mathrm{C}$ in benzene with $n$-butyl-lithium as initiator, and in the presence of a small amount of anisole, as done by Morton, et al.: ${ }^{6}$ Anisole was added in order to enhance the rate of the initiation reaction without giving any influence upon the 1, 4-microstructure of the growing block-chains of butadiene. The initiator was used in a $n$ hexane solution, and the mole ratio of anisole to the initiator was adjusted to 10 for each experiment. In practice, a SB-diblock sample, coded as Di-SB, was synthesized through the following steps. ${ }^{6}$ Benzene was first introduced by distillation at $-78^{\circ} \mathrm{C}$ to a reaction vessel connected to a vacuum system. To this vessel the initiator solution and styrene monomer were added. Immediately after the reaction mixture was thawed, anisole was admixed to it. The polymerization was continued for $5 \mathrm{hr}$. Then 
the coupling reaction of polystyryl anions with butadiene monomers was carried out at least for $12 \mathrm{hr}$, to yield the diblock sample. The preparation procedure of a SBS-triblock sample, coded as Tri-SB, was the same as before, except for a further addition of styrene at the final stage of the aforementioned reaction. To obtain a so-called "tapered" SB-copolymer, coded as Tap-SB, both monomers were simultaneously introduced to the reaction vessel, and the polymerization was made under the same conditions as before, according to the copolymerization kinetics proposed by Wofford and Hsieh. ${ }^{7}$

\section{TLC Procedure}

General descriptions for TLC procedures applicable to polymer separation will be found in our previous papers. ${ }^{8}$ Experimental conditions specific to the present study are noted below. The adsorbents used were Silica Gel B-0 (Wako Chemicals Co., Tokyo) and Alumina Oxide G (E. Merck AG., Darmstadt). The thickness of the thin layers was adjusted throughout to $0.5 \mathrm{~mm}$. Each sample polymer was dissolved in carbon tetrachloride at $c a$. $0.5 \mathrm{~g} / \mathrm{d} l$ to prepare stock solutions. The sample loading onto the chromatoplate was made using freshly prepared stock solutions to avoid the drawback, characteristic of diene polymers, that a substantial amount of the sample remains undeveloped at the starting point. ${ }^{9,10}$ Each sample size was adjusted to an amount between 5 and $10 \mu \mathrm{g}$. Ascending development was made throughout in this study. To some cases, a concentration gradient technique was applied. The experimental details will be given together with the results relevant to each. The visualization of the chromatograms was made by the thymol blue method. ${ }^{11}$

\section{Other Characterization Techniques}

The number-average molecular weight was determined in toluene at $30^{\circ} \mathrm{C}$ with a Knauer Membrane Osmometer (KG Dr. Knauer, Berlin). The styrene content analysis was made in two ways, i.e., by nuclear magnetic resonance and ultraviolet spectroscopy. A Shimadzu Gel Permeation Chromatograph Model 1-A was used for the determination of molecular weight distributions and point-by-point compositions $\langle x\rangle$ at each elution count $(5 \mathrm{~m} l)$, expressed in the styrene content. For the latter purpose, an ultraviolet detector, LKB Uvicord II (LKB, Stockholm), was connected in series to the GPC apparatus. The eluent was THF and the flow rate was adjusted to $1 \mathrm{ml} / \mathrm{min}$.

\section{RESULTS AND DISCUSSION}

\section{Characterization Results}

The results obtained by osmometry, gel permeation chromatography (GPC), and others

Table I. Characteristics of reference and commercial SB-samples tested

\begin{tabular}{|c|c|c|c|c|c|c|}
\hline \multirow{2}{*}{$\begin{array}{l}\text { Samples } \\
\text { code }\end{array}$} & \multirow{2}{*}{$\begin{array}{l}\text { Chain archi- } \\
\text { tecture }\end{array}$} & \multirow{2}{*}{$M_{n} \times 10^{-4}$} & \multirow{2}{*}{$M_{w} / M_{n}^{\mathrm{a}}$} & \multicolumn{2}{|c|}{ ST, wt $\%$} & \multirow{2}{*}{ Producer } \\
\hline & & & & NMR & $\mathrm{UV}(\mathrm{GPC})$ & \\
\hline \multicolumn{7}{|c|}{ Reference samples } \\
\hline Tap-SB & Tapered & 3.7 & 1.09 & 28.1 & $32.5(34)$ & \\
\hline Di-SB & SB-block & 3.4 & 1.13 & 26.4 & $28.5(29)$ & \\
\hline Tri-SB & SBS-block & 3.8 & 1.10 & 29.6 & $32.5(33)$ & \\
\hline RSB-1c & & 14.6 & - & - & 62.4 & \\
\hline RSB-2c & Random & 10.4 & - & - & 38.9 & \\
\hline RSB-3c & & 6.15 & - & - & 23.9 & \\
\hline \multicolumn{7}{|c|}{ Commercial samples } \\
\hline FRS 206 & Random & $4.13^{b}$ & $9.5^{b}$ & - & $31.6(33)$ & Firestone \\
\hline Solprene 300 & Pandom or tanered & $10.4^{b}$ & $2.4^{b}$ & - & $26.5(36)$ & \\
\hline Solprene 301$\}$ & Random or tapered & $12.2^{\mathrm{b}}$ & $1.7^{\mathrm{b}}$ & - & $24.6(30)$ & Phillips \\
\hline Solprene 1205 & SB-block & $5.1^{\mathrm{b}}$ & $1.5^{\mathrm{b}}$ & - & $29.2(32)$ & \\
\hline Kraton 1101 & SBS-block & 7.9 & - & - & $28.7(30)$ & Shell \\
\hline
\end{tabular}

a Determined by GPC

b Data obtained by Kotaka and White, see ref 10 .

c Characterized and supplied by Kotaka. 
Table II. Chain microstructures of butadiene parts in SB-samples ${ }^{\mathrm{a}}$

\begin{tabular}{lccc}
\hline \multirow{2}{*}{$\begin{array}{c}\text { Sample } \\
\text { code }\end{array}$} & \multicolumn{3}{c}{ Microstructure, wt $\%^{\mathrm{b}}$} \\
\cline { 2 - 4 } & $1,2-$-Vinyl & Cis-1, 4 & Trans-1, 4 \\
\hline Tap-SB & 12.5 & 27.7 & 27.3 \\
Di-SB & 12.3 & 25.5 & 33.7 \\
Tri-SB & 11.0 & 30.6 & 25.9 \\
\hline
\end{tabular}

a For the data of commercial products used in this study, see ref 10 .

b Indicated on the basis of wt $\%$ of total polymer.

are listed in Table I, in which data for commercial SB-products tested here and three random SB-copolymers used as the elution standards, which were supplied by Dr. T. Kotaka, are included. Table II shows the chain microstructure of the butadiene parts in our reference samples, which was assessed by an IR-spectroscopic method proposed by Morero, et $a .^{12}$ In Figures $1-3$ are shown GPC elution curves, taken simultaneously with the refractometric (RI) and ultraviolet (UV) detector at $260 \mathrm{~nm}$ for our reference samples. It is interesting to note that the point-by-point composition for the tapered sample, Tap-SB, decreases steeply with increase in elution count, indicating that highermolecular-weight components have higher styrene contents, while those for the diblock and triblock

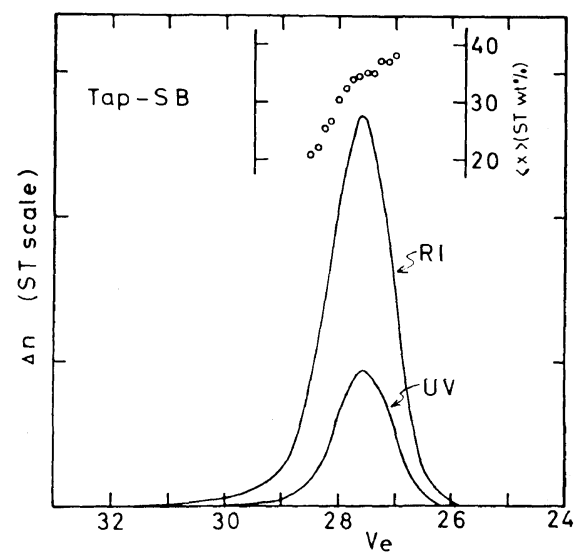

Figure 1. GPC elution curves obtained with refractometric (RI) and ultraviolet (UV) detectors for a SB-sample of tapered type. Open circles indicate plots of point-by-point styrene content against elution count.

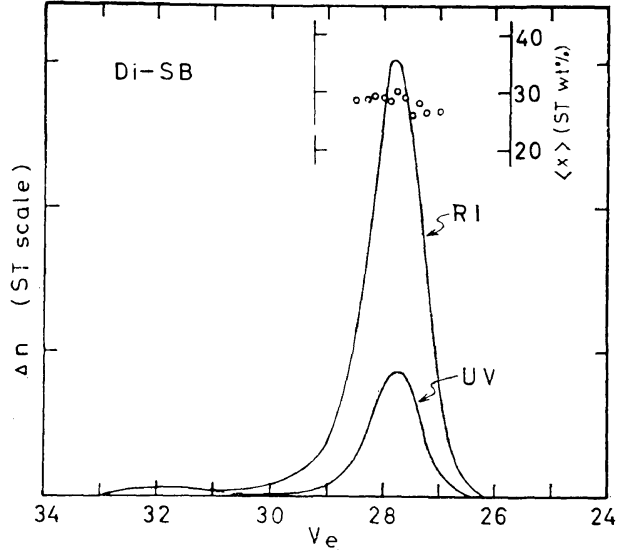

Figure 2. GPC elution curves obtained for a SBdiblock sample, in comparison with Figure 1.

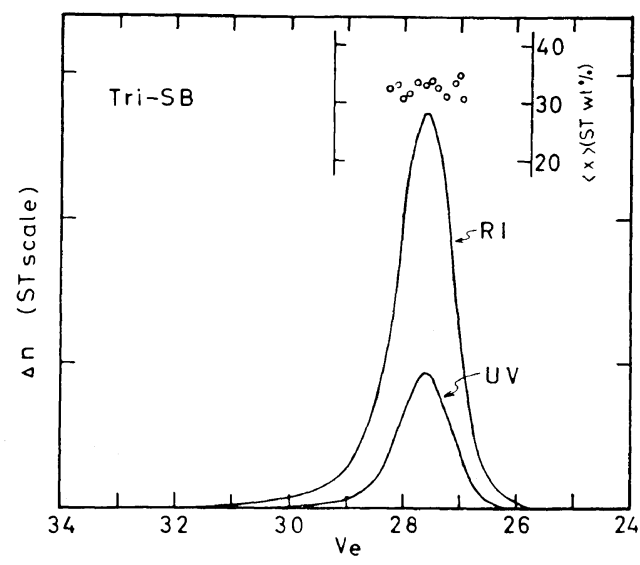

Figure 3. GPC elution curves obtained for a SBStriblock sample, in comparison with Figure 1.

sample show no such trend. The former finding is pertinent to the copolymerization mechanism for SB-copolymers of the tapered type. ${ }^{7}$

Chromatographic Behavior of Reference Samples

Preliminary TLC experiments were carried out to establish the development conditions which would enable us to distinguish our reference samples from one another by the difference in chain architecture. Moderately good and good solvents for both precursor polymers have been used singly as developers. In addition, actions of silica gel and alumina, used as adsorbents, upon polymer separation were investigated, since it is presumed that the diene structure exerts many more specific interactions with the latter 
Table III. $R f$ values for reference SB-samples obtained with single solvents as developer

\begin{tabular}{|c|c|c|c|c|c|c|c|c|c|c|c|c|}
\hline \multirow[b]{2}{*}{ Solvent } & \multirow{2}{*}{$\begin{array}{l}\text { Dielec. } \\
\text { const. }\end{array}$} & \multirow[b]{2}{*}{$\varepsilon^{0 \mathrm{a}}$} & \multicolumn{5}{|c|}{$R f$ for silica } & \multicolumn{5}{|c|}{$R f$ for alumina } \\
\hline & & & PS & PB & $\begin{array}{c}\text { Tap- } \\
\text { SB }\end{array}$ & Di- & Tri- & PS & $\mathrm{PB}$ & Tap- & Di- & $\begin{array}{l}\text { Tri- } \\
\text { SB }\end{array}$ \\
\hline Cyclohexane & 2.02 & 0.04 & 0 & 0 & 0 & 0 & 0 & 0 & 0 & 0 & 0 & 0 \\
\hline $\mathrm{CCl}_{4}$ & 2.24 & 0.18 & 0 & $1^{\mathrm{b}}$ & 0 & 0 & 0 & 0 & $1^{\mathrm{b}}$ & 1 & 1 & 0 \\
\hline Amyl chloride & 6.6 & 0.26 & 0 & $1^{\mathrm{b}}$ & 1 & 1 & 1 & 1 & $1^{\mathrm{b}}$ & 1 & 1 & 1 \\
\hline$\rho$-Xylene & 2.27 & 0.26 & 1 & 1 & 1 & 1 & 1 & 1 & 1 & 1 & 1 & 1 \\
\hline Benzene & 2.28 & 0.32 & 1 & 1 & 1 & 1 & 1 & 1 & 1 & 1 & 1 & 1 \\
\hline $\mathrm{CH}_{3} \mathrm{Cl}$ & 4.31 & 0.40 & 1 & 1 & 1 & 1 & 1 & 1 & 1 & 1 & 1 & 1 \\
\hline THF & 7.42 & 0.45 & 1 & 1 & 1 & 1 & 1 & 1 & 1 & 1 & 1 & 1 \\
\hline
\end{tabular}

a Solvent strength parameter defined by Snyder, see ref 13 .

b $R f$ changes from zero to unity according to the microstructure, see ref 9 .

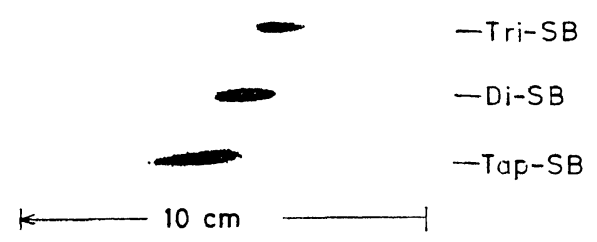

Figure 4. Chromatograms obtained by adsorption mechanism for reference SB-samples of tapered, diblock, and triblock type.

adsorbent than with the former. ${ }^{13}$ The results are summarized in Table III, which indicates that no single solvent can give different $R f$ values for different polymer species, except for carbon tetrachloride; further study using this solvent and alumina will be described later.

At the next stage we chromatographed our reference samples by a concentration gradient technique $^{8}$ with silica gel thin-layers, activated at $120^{\circ} \mathrm{C}$ for $2 \mathrm{hr}$. For the development, a binary cyclohexane-chloroform ( $9: 1$ by volume) and chloroform were used as the initial and adding solvent, respectively. A chromatogram was obtained as shown in Figure 4, which proves that it is feasible to distinguish the triblock sample from either the diblock or the tapered sample. However, the difference among the $R f$ values was not large enough for a perfect separation, and the tailing phenomena appearing in the chromatogram could not be suppressed completely. A feature specific to this chromatogram was that the diblock sample migrated faster than the triblock sample, which is just the opposite to the case of SM-block copolymers. ${ }^{2}$
The reason for this is still open for discussion.

\section{Chain Architecture of Commercial Products}

Referring to the foregoing result, we attempted chromatographically to identify the chain architecture of four commercial products (see Table I). Almost the same gradient technique was applied. In practice, the composition and volume of the binary cyclohexane-chloroform used as the initial solvent were $15: 1$ by volume and $160 \mathrm{ml}$, respectively. To this solvent, $80 \mathrm{ml}$ of chloroform was added at a constant rate during the development. In this experiment, our diblock and tapered sample, a narrow distribution polystyrene (PS), a commercial polybutadiene (Diene 55) and a commercial SBproduct of random type (FRS 206) were chromatographed simultaneously. The $R f$ values thus found for PS, FRS 206, and Diene 55 were $0.23,0.56$, and 0.78 , respectively, whereas those for our tapered and diblock samples, Solprene 300, 301, and 1205, and Kraton 1101 were hardly distinguishable, ranging between 0.33 and 0.39 . This suggests that separation by the difference in chain architecture is practically impossible under this experimental condition.

Recalling that TLC developments with single solvents or mixtures often result in better separation than do those by gradient techniques, ${ }^{14}$ we chromatographed the commercial samples simply by using the same binary as above but having a fixed composition, i.e., $140: 75$ by volume. The chromatogram is shown in Figure 5 . Apparently two commercial products, Solprene 300 and 301 can be identified as tapered types, 


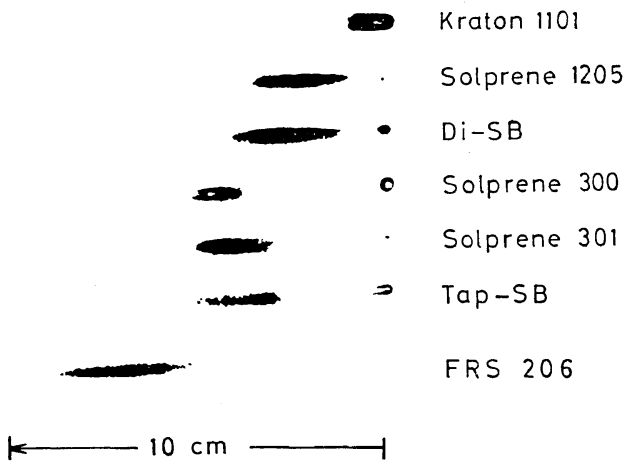

Figure 5. Chromatograms obtained by adsorption mechanism for commercial SB-products and reference samples

while Solprene 1205, and Kraton 1101 appear as SB-diblock and SBS-triblock types, respectively. The identifications are in ageement with those given by each manufacturer, though Solprene 300 and 301 are both described as random type by the manufacturer.

The above finding was further justified by a series of additional experiments. One of them was to investigate the composition dependence of $R f$ under the present development conditions, since the compositions of the samples under test were slightly different from one another. Three random SB-copolymer samples having different compositions, RSB-1, -2 , and -3 , were subjected to the same TLC experiment as before,

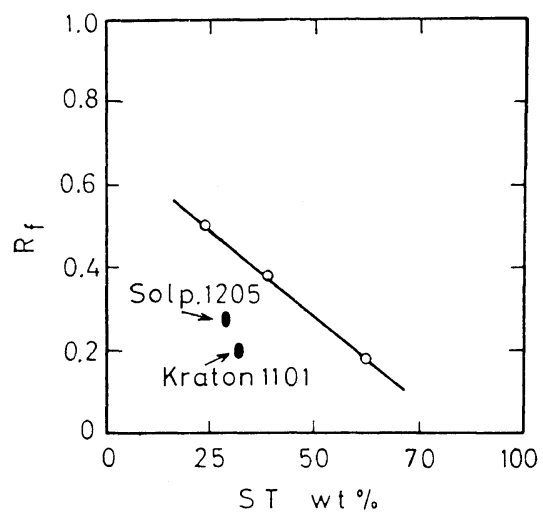

Figure 6. Plots of $R f$ against the styrene content for random SB-copolymers (O), Solprene 1205 (O), and Kraton $1101(\bullet)$, found under the same experimental conditions as those for Figure 5. together with Solprene 1205 and Kraton 1101. The result is illustrated by Figure 6, in which $R f$ values are plotted against the styrene content. This implies that the difference in composition among the commercial products will exert no substantial influence upon such chromatographic behavior as shown in Figure 5.

Component Species Separated from Kraton 1101

During the course of the GPC study on the commercial products we found that Kraton 1101 showed a little different elution behavior from the other products. Figure 7 shows the result obtained for this product by the dual-detector GPC. As seen in the figure, each elution curve exhibits three peaks, at elution counts $27.3,29.0$, and 33.0; these will be denoted as $P(\mathrm{a}), P(\mathrm{~b})$, and $P(\mathrm{c})$, respectively. The peaks $P(\mathrm{a})$ and $P(\mathrm{c})$ may be assigned immediately to the main

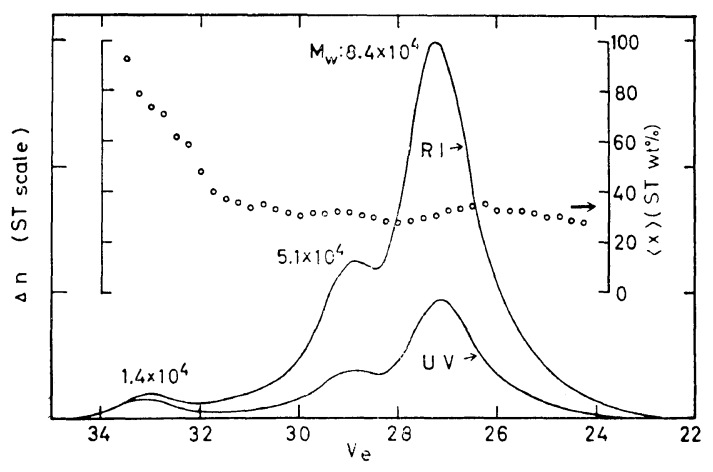

Figure 7. GPC elution curves obtained with refractometric (RI) and ultraviolet (UV) detectors for a commercial SB-product, Kraton 1101. Open circles indicate plots of point-by-point styrene content against elution count.

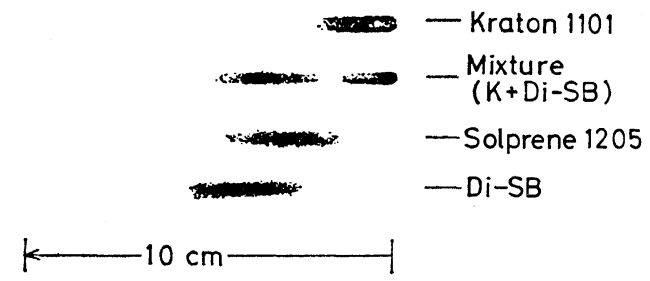

Figure 8. Chromatograms obtained by adsorption mechanism for Kraton 1101, Di-SB, an equiponderant mixture of the former two, and Sloprene 1205 . 
component species of this product and the occluded homopolystyrene, respectively, by referring to the point-by-point compositions, $\langle x\rangle$, indicated by open circles in the figure. However, the assignment for the component species appearing at $P(b)$ cannot be made, except for its composition, which is nearly the same as the main component species. To explore the chain architecture of this unknown species, we chromatographed Kraton 1101 and its equiponderant mixture with our diblock sample under the same condition as applied to obtain Figure 5. The result is shown in Figure 8. Kraton 1101 shows a similar chromatographic behavior to that of our triblock sample, and there is no evidence that it contains any diblockcomponent species.

The above result was obtained by the polaritycontrolled adsorption mechanism. ${ }^{3}$ To confirm the result further we have applied another separation mechanism, i.e., the solubilitycontrolled phase-separation mechanism, ${ }^{3}$ which leads to fractionation largely by molecular weight. A gradient development on silica gel thin-layer was performed at $c a .20^{\circ} \mathrm{C}: 100 \mathrm{~m} l$ of a $3: 2$ binary of chloroform and methanol was placed in a development chamber as the initial solvent, and, during the development, $80 \mathrm{ml}$ of methanol was added at a constant rate. Figure 9 shows the chromatogram, on which Kraton 1101 is separated into three components, though in the figure, the fastest component, which shows $R f=0.85$, is too faint to be visualized by staining. Apparently the fastest, the faster component, and the slow component should

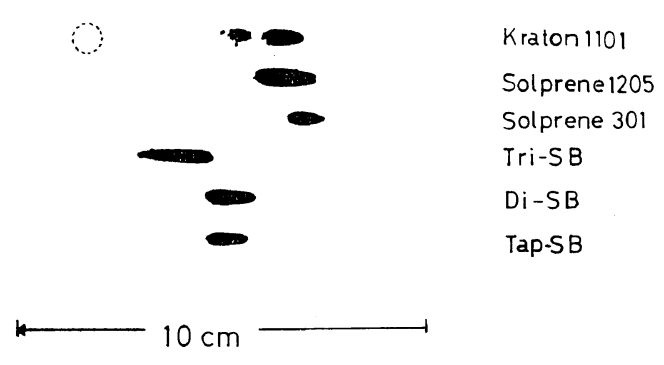

Figure 9. Chromatograms obtained by phaseseparation mechanism for Kraton 1101 and others. A dotted circle indicates the final spot for the fastest component separated from Kraton 1101.

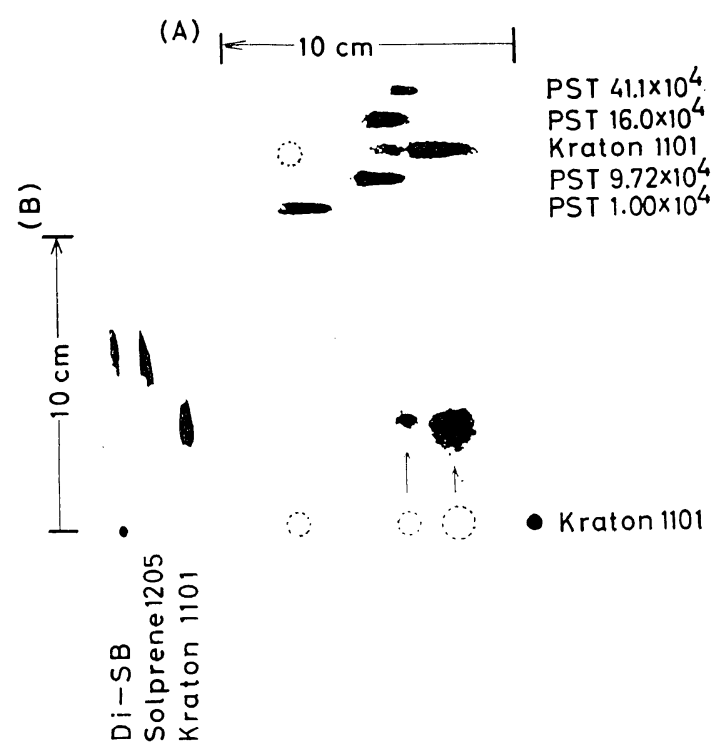

Figure 10. Two-dimensional chromatograms prepared to determine the chain architecture of Kraton 1101. For details, see text. In the reference chromatogram, the numbers attached to PST(polystyrene) indicate molecular weights.

correspond to those appearing at $P(\mathrm{a}), P(\mathrm{~b})$, and $P(\mathrm{c})$ in the GPC curve, respectively.

Our next idea was to apply a two-dimensional TLC development technique to identify the chain architecture of the faster component separated as above. The experimental procedure has been described elsewhere. ${ }^{9}$ Figure 10 shows the chromatogram thus obtained. As is seen in the figure, Kraton 1101 was spotted on the left lower corner of the chromatoplate to prepare the main chromatogram. Two reference chromatograms were prepared, one on the rightside and the other on the upper left-hand corner of the chromatoplate. After Kraton 1101 had been separated by molecular weight (the phase separation mechanism) in the direction (A), the secondary development perpendicular to (A), denoted as (B), was carried out by the adsorption mechanism, i.e., in the same manner as that used to obtain Figure 8. By comparing the main chromatogram with the reference ones, it may be justified again that Kraton 1101 does not contain any diblock component. Hence, we are inclined to conclude that Kraton 1101 is composed of two triblock components having 
nearly the same composition but different molecular weights, though this conclusion is hardly conceivable from the polymerization mechanism. In this connection we may add that the component species at $P(b)$ cannot be of a radial teleblock type, ${ }^{4}$ judging from the elution count at which $P(b)$ appeared.

Use of Alumina as Adsorbent

The last topic concerns the action of alumina upon separation. As is shown in Table III, the TLC system composed of alumina and carbon tetrachloride indicated complete separation of triblock from diblock and also from tapered SB-copolymers. This system was again applied to the commercial products. However, the chromatographic distinction among the random, tapered, and diblock products was again impossible by this procedure, so we have searched for another appropriate situation in which to use alumina as adsorbent. After a number of TLC runs using various solvent combinations, we found that a binary carbon tetrachloride$n$-hexane $(9: 1$ by volume) fulfilled our requirement reasonbly well: the tapered sample migrated up to the solvent front and the diblock sample showed a long upward tailing, whereas the triblock sample remained immobile. As this experimental condition implies, a prerequisite for good separation attained with alumina is that the developer system is composed of solvents of lower polarities than carbon tetrachloride. Another condition is to use highly activated alumina. Since this condition is quite sensitive to humidity, it should be kept in mind that the reproducibility of separations using alumina is not high enough to allow routine work.

Acknowledgments. The authors wish to thank Prof. T. Kotaka, this Institute, for his kindness in supplying the samples and his valuable suggestions. The research herein reported was performed pursuant to a research grant to this Institute by the International Institute of Synthetic Rubber Producers, Inc.

\section{REFERENCES}

1. F. Kamiyama, H. Matsuda, and H. Inagaki, Makromol. Chem., 125, 286 (1969).

2. Kotaka, T. Uda, T. Fukuda-Tanaka, and H. Inagaki, ibid., 176, 1273 (1975).

3. H. Inagaki, F. Kamiyama, and T. Yagi, Macromolecules, 4, 133 (1971); F. Kamiyama and H. Inagaki, Bull. Inst. Chem. Res., Kyoto Univ., 49, 53 (1971).

4. See, e.g., J. R. Haws, and T.C. Middlebrook, Rubber World, 167 (4), 27 (1973).

5. L. J. Fetters, J. Polym. Sci., Part C, 26, 1 (1969).

6. M. Morton, J. E. McGrath, and P. C. Juliano, ibid., Part C, 26, 99 (1969).

7. C. F. Wofford and H. L. Hsieh, ibid., Part $A-1$, 7, 461 (1969).

8. H. Inagaki H. Matsuda, and F. Kamiyama, Macromolecules, 1, 520 (1968); T. Miyamoto, Kobunshi, 23, 378 (1974).

9. N. Donkai, N. Murayama, T. Miyamoto, and H. Inagaki, Makromol. Chem., 175, 187 (1974).

10. T. Kotaka and J. L. White, Macromolecules, 7, 106 (1974)

11. F. Kamiyama, H. Matsuda, and H. Inagaki, Polymer J., 1, 518 (1970).

12. D. Morero, A. Santabrogio, L. Porri, and F. Ciampelli, Chim. Ind. [Milano] 41, 758 (1959).

13. L. R. Snyder, "Principles of Adsorption Chromatography," Marcel Dekker, New York, N. Y., 1968.

14. H. Inagaki and F. Kamiyama, Macromolecules, 6, 107 (1973); F. Kamiyama and H. Inagaki, Bull. Inst. Chem. Res., Kyoto Univ., 52, 398 (1974). 